\title{
Huge boost for genetics of cognitive disorders
}

A large-scale study has identified 50 new candidate genes that may be involved in autosomal-recessive forms of intellectual disability in humans almost doubling the number of genes implicated in non-syndromic intellectual disability and highlighting the power of next-generation sequencing to elucidate the molecular genetic basis of a complex disorder.

Most genetic research into cognitive impairment has focused on X-chromosome-linked forms of intellectual disability; although these studies have uncovered $>90$ pathogenic gene mutations, $\sim 60 \%$ of intellectual disability cases still have no known genetic basis. The authors therefore decided to focus on autosomal-recessive forms of intellectual disability, choosing as study subjects 136 consanguineous families from Iran who are affected by moderate or severe forms of intellectual disability.

Identifying causal variants by sequencing or candidate-gene approaches is laborious and costly. However, the process has recently been made easier and more costeffective by the advent of exome sequencing, which combines exome capture and next-generation sequencing. For this project, the exome sequencing protocol was made more efficient by narrowing down the targeted regions to exons that lay in homozygous intervals.

Single causal mutations were found in homozygous form in 78 of the 136 families: these include 23 variants with known involvement in intellectual disability or related neurological disorders, as well as 50 that had not previously been linked to intellectual disability. Some of the new candidate genes encode neuron- or brain-specific functions, such as glial differentiation or synaptic transmission. However, many more of them have 'housekeeping' functions and are primarily involved in histone function and in the regulation of gene transcription and translation.

Only two of the new genes were mutated in more than one family, confirming the heterogeneous nature of intellectual disability. Although some of the identified variants might be false positives - for some mutations, no obvious link can be made between the molecular defect and intellectual disability - it is encouraging that so many of the new candidates interact with established intellectual disability genes in protein and regulatory networks. The more-promising candidates will hopefully be confirmed by functional studies, which might also contribute to understanding how ubiquitously expressed genes can lead to brain-specific phenotypes.

Tanita Casci

ORIGINAL RESEARCH PAPER Najmabadi, $\mathrm{H}$. et al. Deep sequencing reveals 50 novel genes for recessive cognitive disorders. Nature 21 Sep 2011 (doi:10.1038/nature10423)

FURTHER READING Bamshad, M. J. et al.

Exome sequencing as a tool for Mendelian disease gene discovery. Nature Rev. Genet. 27 Sep 2011 (doi:10.1038/nrg3031)

Transcriptional and translational network showing the relationship between protein complexes (neutral), new autosomalrecessive intellectual disability proteins (red), and known intellectual disability proteins (blue). Arrows indicate post-translational modifications. Image is modified, with permission, from Najmabadi, H. et al. (c) (2011) Macmillan Publishers Ltd. All rights reserved.

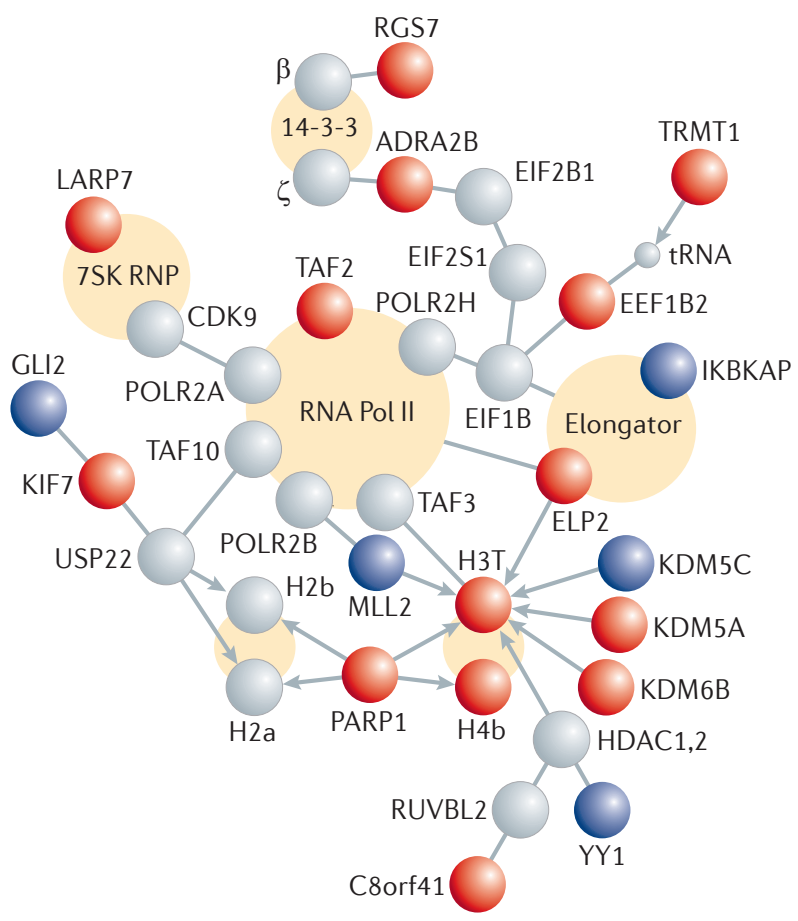

